\title{
L'éducation à la citoyenneté : une responsabilité désormais explicite à l'école québécoise d'accord, mais au nom de quelles valeurs ?
}

\author{
France Jutras (University of Sherbrooke)
}

Résumé. Partant d'une analyse des visées de l'éducation à la citoyenneté dans les discours des organismes mondiaux voués à la promotion de l'éducation, de la politique éducative québécoise et des programmes d'études du primaire et du secondaire qui l'actualisent, ainsi que de la recherche sur les buts et les moyens mis à la disposition du personnel enseignant pour l'intervention en ce sens, cet article met en relief les grandes orientations de l'éducation à la citoyenneté de manière à dégager ses valeurs fondatrices. Puis, une étude empirique auprès de novices en enseignement au secondaire permet d'identifier les valeurs que ceux-ci disent vivre dans leur pratique quotidienne d'éducation et de les mettre en relation avec les valeurs des grandes orientations de l'éducation à la citoyenneté.

Abstract. Starting with an analysis of the goals of citizenship education, as seen in the discourses of world organizations devoted to the promotion of education, Québec educational policy, elementary and secondary programs of study, as well as research on the goals and means available to teaching personnel in this domain, this paper dwells upon the major orientations of citizenship education so as to bring out its founding values. Then, an empirical study of beginning secondary teachers, permits an identification of the values reportedly lived in their daily educational practices and their linkage to the major orientations of citizenship education.

\section{L'école interpellée à la participation citoyenne}

Avec $60,5 \%$ d'électeurs inscrits qui ont exercé leur droit de vote aux élections de juin 2004, le Canada a connu sa plus faible participation aux élections fédérales depuis 1979, alors que le taux avait été de 75,7\% (Munger, 2004). Politologues et sociologues émettent diverses hypothèses qui visent à expliquer la chute du taux de participation aux élections. Mais, quelle que soit l'explication, de façon unanime, ils estiment que l'éducation représente l'une des solutions pour inciter la population à aller voter, pour qu'elle s'intéresse au lien entre la politique et la vie quotidienne. Ce genre de remarque revient à chaque élection car on considère que le manque d'intérêt pour la chose politique nuit à la vie démocratique. Et on attend de l'école qu'elle participe à la formation du citoyen. Oui l'école québécoise est interpellée. Nous allons voir en quel sens. Oui les enseignantes et enseignants le sont aussi. Nous allons voir pourquoi et ce qui est attendu d'eux. Mais surtout nous allons tenter de dégager les valeurs qui fondent l'éducation à la citoyenneté et porter un regard sur des valeurs qu'enseignantes et enseignants disent actualiser dans leur pratique pédagogique.

12 Canadian and International Education Vol. 34 no. 1 - June 2005 


\section{L'intégration de l'éducation à la citoyenneté à l'école}

À partir des années 1980, principalement dans les pays à démocratie libérale, les enjeux du développement de la personne comme être social, se sont cristallisés autour de la question de l'éducation à la citoyenneté. Plusieurs caractéristiques actuelles de la vie en société sont à la base de la nécessité de faire de l'éducation à la citoyenneté (Pagé, 2001; Soares, 2004) : la composition multiculturelle et multiraciale de la population, la place des identités minoritaires, l'égalité des droits; la violence, le vandalisme et la criminalité qui provoquent des sentiments d'insécurité; la remise en cause des structures de l'État providence dans le cadre de politiques néolibérales qui réduisent le rôle de l'État; le vide politique qui s'exprime par la perte de crédibilité des partis politiques auprès des électeurs. Cet état de fait pose évidemment de nouveaux défis sociaux qu'on demande au corps enseignant et au monde de l'éducation dans son ensemble de relever. C'est ainsi que non seulement au Canada, mais partout dans le monde, les enseignants sont désormais fortement interpellés pour apporter une contribution significative à la formation sociale de la personne afin de lui fournir les repères et les balises nécessaires à une vie partagée avec autrui dans l'espace public. Dès 1996, le Rapport Delors (Unesco, 1996) a souligné que les systèmes éducatifs ne pouvaient plus se contenter de considérer la personne dans sa seule individualité, mais qu'ils devaient au contraire insister fortement sur sa dimension sociale et politique. Aussi, aux trois piliers traditionnels de l'éducation - apprendre à connaître, à faire et à être -, a-t-il recommandé d'en ajouter un quatrième : apprendre à vivre ensemble. Il s'agit d'une visée éducative différente : elle dépasse la finalité du développement de l'individu pour lui-même et elle concerne le développement de sentiments d'appartenance, de la volonté et de la capacité d'agir avec les autres dans un monde où tout est interconnecté et interdépendant. Son omission, d'après le Bureau international de l'éducation (2003), pourrait avoir comme conséquence l'annihilation de tous les autres efforts en faveur de l'éducation, de la santé et du développement.

Ces besoins nouveaux de formation, de même que les recommandations des États généraux sur l'éducation (1996) ont amené le Gouvernement du Québec à élaborer une nouvelle politique éducative en 1997, Le virage du succès, qui a donné lieu à des changements substantiels dans la Loi sur l'instruction publique en 1998 et à la réforme de l'école québécoise mise en œuvre graduellement depuis 2000. On peut remarquer que les politiques de l'éducation au Québec concordent avec les orientations de l'Unesco. Prenons les trois missions de l'école : instruire, socialiser et qualifier. L'accent est mis sur l'apprentissage de connaissances, de savoir-être et de savoir-faire qui devraient permettre de se préparer à une insertion sociale où on apporte sa contribution de travailleur et de citoyen. On va parler, dans le discours ministériel et dans celui du Conseil supérieur de l'éducation (1998), de lutter contre l'exclusion et la violence et de prendre conscience de la diversité et des interdépendances dans le monde, ainsi 
que du pluralisme de notre société. Le besoin de faire de l'éducation à la citoyenneté à l'école y est donc explicitement affirmé. L'intégration de l'éducation à la citoyenneté au curriculum scolaire québécois prend deux formes. Premièrement, elle constitue maintenant une composante des programmes d'études. Ainsi, au préscolaire et au primaire, les programmes de Géographie, histoire et éducation à la citoyenneté portent sur la société et le territoire; au secondaire, ces questions sont traitées dans les programmes d'Histoire et éducation à la citoyenneté. Deuxièmement, le thème du "vivreensemble et citoyenneté » compose l'un des cinq domaines généraux de formation qui sont transversaux et qui sont censés être intégrés aux diverses matières afin de développer l'esprit critique au préscolaire, au primaire et au secondaire. L'intention éducative qui sous-tend les domaines généraux de formation dans le curriculum est de conduire l'élève à «établir des liens entre leurs apprentissages scolaires, les situations de leur vie quotidienne et les phénomènes sociaux » (MEQ, 2003, p. 21).

Ainsi, avec la réforme, l'éducation à la citoyenneté est devenue la responsabilité explicite de tout le personnel enseignant de l'école. Que doit comprendre l'éducation à la citoyenneté ? Il se dégage des écrits sur le sujet que ses contenus portent sur la connaissance et la compréhension des droits de la personne, de l'histoire nationale, des structures gouvernementales et des processus de la vie politique; ses méthodes et ses moyens visent des apprentissages actifs qui mettent l'élève en situation de pratiquer la citoyenneté et d'en débattre à l'école et dans la communauté; ses buts consistent à outiller les élèves par des connaissances, de la compréhension, des habiletés et des aptitudes, des valeurs et des dispositions pour qu'ils puissent participer de manière active, responsable et critique à la vie sociale démocratique. De plus, ce qu'on appelait encore récemment l'éducation interculturelle, multiculturelle et antiraciste est maintenant intégré aux visées de l'éducation à la citoyenneté. En bref, l'éducation à la citoyenneté est vue comme un levier de régulation des rapports sociaux. Bien qu'elle ne devrait guère être limitée à l'école, ici nous voulons comprendre le rôle spécifique de l'école à cet égard tel qu'il est actualisé par le travail quotidien du personnel enseignant.

\section{La tâche du personnel enseignant par rapport à l'éducation à la citoyenneté}

On trouve dans les écrits de nombreuses descriptions et recommandations au sujet de la manière de faire l'éducation à la citoyenneté. Cependant, la tâche concrète du personnel enseignant a moins été l'objet d'analyse. Meirieu (1997), par exemple, soulève que le pluralisme des valeurs dans nos sociétés la rend difficile car les enseignants ne savent plus à quelles valeurs éduquer les jeunes qui leur sont confiés. Cela est d'autant plus évident qu'éducation et politique se rejoignent dans le cas de l'éducation à la citoyenneté, ce qui apparaît plein de risques et d'embûches pour le corps enseignant. Dans ces circonstances, il préfère trop souvent se retrancher dans un enseignement formel, descriptif et 
abstrait (Osler et Starkey, 1997). D'ailleurs, plusieurs études confirment le fait que l'enseignement de sujets délicats et d'enjeux controversés est une tâche que les enseignants souhaitent ne pas avoir à accomplir. Pour le moins qu'on puisse dire, ces questions renvoient au rôle de l'école et au rôle très spécifique des enseignants, au-delà de l'enseignement des matières scolaires, dans la visée de la formation morale et sociale de la personne en tant que citoyenne ou citoyen d'une démocratie libérale.

Pour faire le point sur l'état de la situation internationale de l'éducation à la citoyenneté dans les écoles, ne pouvant s'appuyer sur des études empiriques, Mintrop (2002) a eu recours à des analyses de documents officiels et de matériel pédagogique, des synthèses de discussions, des rapports d'experts en éducation à la citoyenneté et d'administrateurs scolaires. Il conclut à une grande faiblesse des connaissances et du rapport au savoir des enseignants chargés de faire l'éducation à la citoyenneté à partir des éléments suivants : les enseignants ne sont pas des spécialistes du domaine, leur savoir n'est pas constitué d'un corps de connaissances formalisées, mais plutôt d'un savoir informel, personnel, appris par expérience de vie, de sens commun et peu réfléchi. Plus inquiétant encore, Mintrop rapporte que malgré les avis des experts sur les limites du savoir des enseignants, ceux-ci ne considèrent pas avoir besoin de formation continue en éducation à la citoyenneté. Cependant, pour le Québec, Guay et Jutras (2004) rapportent les résultats de deux enquêtes réalisées en 2001 et 2002 auprès de toutes les commissions scolaires à l'effet que la demande de formation continue dans ce domaine est importante. Le personnel enseignant du Québec considère en effet disposer de peu de moyens pour faire l'éducation à la citoyenneté et ne pas connaître véritablement le sens et les enjeux de celle-ci.

Il n'en demeure pas moins qu'avec la réforme de l'éducation au Québec, au-delà de leur enseignement disciplinaire - que ce soit en langues, en mathématiques, sciences et technologies, en arts, en développement personnel ou en univers social -, les enseignants partagent des responsabilités éducatives communes dans le cadre des domaines généraux de formation. Les visées de formation de ces domaines peuvent, selon le Ministère (2003), être mises en œuvre à l'intérieur de la classe, dans le cadre de la discipline enseignée en faisant appel à des ouvertures interdisciplinaires et multiniveaux, et dans la vie de l'école dans le cadre du projet éducatif, des activités parascolaires et d'actions ponctuelles. Pour ce qui concerne spécifiquement le domaine général du vivre-ensemble et citoyenneté, c'est encore une fois autant dans les classes que dans l'ensemble de l'expérience à l'école que se présentent les occasions de formation de la citoyenne ou du citoyen. Les buts éducatifs identifiés par le Ministère (2003) dans le domaine du vivre-ensemble et citoyenneté ne sont pas étrangers à des pratiques déjà plutôt bien implantées dans les écoles : la valorisation des règles de vie en société et des institutions démocratiques; l'engagement, la coopération et la solidarité; la contribution à la culture de paix. Rares sont les disciplines où des questions morales et sociales qui ne sont pas débattues, où l'organisation et la gestion de classe ne peuvent donner lieu à des approches d'inclusion qui 
permettent d'expérimenter des comportements citoyens et des sentiments d'appartenance. Rares sont les écoles secondaires où les élèves n'élisent pas de représentants pour participer à des instances administratives et de gestion. Or, réforme ou non, domaine général de formation ou non, depuis longtemps déjà, les interventions visant la socialisation des élèves font partie du travail enseignant. Car, sans un minimum de valeurs partagées et respectées du vivreensemble, aucune classe, aucun enseignement, aucun apprentissage n'est possible. Avec la réforme, ces visées deviennent plus explicites et elles clarifient le fil conducteur du développement social de la personne dans son expérience scolaire.

\section{Les valeurs de l'éducation à la citoyenneté}

Jusqu'à maintenant nous avons vu d'une part que la sphère politique, au moyen des politiques éducatives et des programmes d'études, transmet au terrain et aux agents éducatifs un ensemble de prescriptions concernant les visées socioéducatives à actualiser auprès des personnes en développement que sont les élèves. La visée du développement de la citoyenneté à l'école par l'école est maintenant intégrée de manière très explicite aux programmes d'étude et au mandat du personnel enseignant avec la réforme de l'éducation. D'autre part, un constat s'impose cependant : autant dans la littérature scientifique que dans les discours des enseignants, il se dégage le problème du manque de connaissances et de savoir-faire pour remplir leurs responsabilités éducatives actuelles. Mais comme Monsieur Jourdain faisait de la prose sans le savoir, les enseignants font depuis longtemps œuvre de socialisation auprès des élèves dans leurs interventions. En effet, pour que la classe fonctionne, il faut une internalisation d'un ensemble de règles d'appartenance et de vivre-ensemble. D'ailleurs, les enseignants qui ont participé à une table ronde sur l'éducation à la citoyenneté rapportée par Brossard (1998) sont unanimes pour dire que l'éducation à la citoyenneté est une éducation aux valeurs et qu'ils en font au quotidien du simple fait qu'ils sont des éducateurs au sens large du terme, et pas seulement des enseignantes ou enseignants de matières scolaires. Cela nous conduit à examiner, au-delà du discours ministériel et du discours sur les enseignants, les finalités et les valeurs de l'éducation à la citoyenneté à l'école.

On peut dire que la réflexion philosophique sur l'éducation à la citoyenneté remonte aux philosophes de la Grèce antique. Ils tenaient pour l'une des finalités de l'éducation de faire de l'individu un citoyen, c'est-à-dire de l'intégrer à la polis, à la cité. Le citoyen pouvait alors participer aux débats et aux affaires de la cité, et même se battre pour elle lorsque cela était requis. Bien sûr, maintenant nous sommes forcément très éloignés de l'antique cité grecque car notre société est immense, complexe et ouverte. Pourtant la mission sociale de l'éducation demeure. Mais aujourd'hui, contrairement à la cité grecque qui comprenait des milliers de citoyens, nos concitoyens se comptent par millions. De plus, dans une même société, on observe la coexistence de cultures différentes, de valeurs 
différentes, de dieux différents. Et finalement, nos appartenances sont multiples: du plus local au plus global. Quelles sont alors les valeurs de l'éducation à la citoyenneté aujourd'hui ?

S'aventurer à répondre à une telle question peut sembler téméraire. Mais cela s'avère absolument essentiel. Car on n'ose le faire ni dans la documentation ministérielle, ni dans la réflexion sur la pratique pédagogique. Dans notre société pluraliste, où le politiquement correct est de rigueur sous prétexte de ne pas heurter des minorités, aucune voix ne s'élève pour mettre en évidence les valeurs profondes qui devraient nourrir et soutenir le projet ministériel d'éducation à la citoyenneté et le travail du personnel enseignant qui doit l'actualiser. Il est certes affirmé en maints endroits que l'éducation à la citoyenneté doit aller au-delà des explications sur le fonctionnement et les raisons d'être des institutions démocratiques. Elle doit être une formation de la personne non seulement au plan intellectuel, mais aussi aux plans affectif et social, c'est-à-dire une éducation aux valeurs. Mais, si nulle part on ne clarifie les valeurs à promouvoir et les raisons pour lesquelles elles doivent être actualisées à l'école, comment pensons-nous que le personnel enseignant sera à même de jouer son rôle à l'égard de l'éducation à la citoyenneté ? D'autant plus qu'enseignantes et enseignants se plaignent régulièrement que les valeurs mises de l'avant généralement par l'école vont à contre-courant des valeurs sociales (Jutras et Boudreau, 1997): l'école prône la coopération alors que la compétition est omniprésente dans notre monde; l'éducation vise le développement de la personne dans le long terme alors que c'est toujours la performance immédiate qui est valorisée; l'école fait la promotion de valeurs qui sont à la baisse dans la société : la stabilité, le respect des personnes, la politesse, le respect de l'autorité.

Pour dégager les valeurs qui animent le projet de faire l'éducation à la citoyenneté aujourd'hui, nous allons mettre en évidence un ensemble d'orientations à propos de celle-ci qui font consensus dans les sociétés occidentales parmi les penseurs et chercheurs du domaine. Une première orientation recouvre les droits de la personne en tant qu'individu : dignité de la personne, égalité devant la loi, égalité hommes-femmes, droit à la dissidence, pluralisme religieux. Une deuxième orientation porte encore sur la dimension des droits, mais cette fois il s'agit des droits de la personne en tant que citoyen : droits à la santé, à l'éducation, au bien-être, droit d'être informé, de faire partie d'une association, de participer aux décisions relatives à sa société. Une troisième met de l'avant l'importance de tisser et de consolider le lien social et le vivre-ensemble. Ainsi, la participation à une culture commune ou à l'appartenance à un même espace public est censée favoriser l'émergence de la solidarité entre les personnes. Cela permet d'identifier un écueil : l'exclusion qui entraîne la perte de l'appartenance et la perte du lien social. Enfin, une quatrième orientation pose la nécessité de la démocratie et de la participation à la vie démocratique et politique, notamment avec le droit de vote, la participation à la critique sociale et la recherche de justice sociale. C'est ainsi 
que se révèle toute la tension qui peut exister entre la sphère privée reliée à la liberté individuelle et le bien commun avec son idéal de résolution de conflits par la discussion plutôt que par des affrontements violents. En dépit des divers modèles de citoyenneté qui existent, les citoyens sont interpellés autant pour ce qui concerne leur village natal que le village global. Mais tout cela ne devrait pas occulter la préoccupation par rapport à la diversité des appartenances dans un pays d'immigration comme le nôtre : comment situer la dialectique entre le civique et l'ethnique ? Faut-il transcender l'ethnique par le civique ou s'arracher de l'ethnique par le civique?

Ces orientations, issues d'une analyse de travaux de sciences humaines et de sciences de l'éducation, illustrent bien les priorités qu'on attribue généralement à l'éducation à la citoyenneté : droits de la personne comme individu, comme citoyen, lien social, démocratie et participation à la vie démocratique. Ces orientations fournissent des indications du but poursuivi et pointent vers des valeurs fondamentales de l'éducation à la citoyenneté. Lesquelles ? Si les orientations nous donnent le sens poursuivi par l'éducation à la citoyenneté, les valeurs qui s'y rattachent devraient nous informer de l'idéal au nom duquel on agit en ce sens. Car les valeurs sont toujours liées à un bien et à un mieux, pense Reboul (1989). En effet, éduquer ou apprendre c'est toujours viser un plus. Par exemple, quand on apprend à écrire, on apprend à bien écrire, ou du moins l'enseignante vise que son élève apprenne à bien écrire. Une valeur, c'est donc un idéal vers lequel on tend ou encore un référent au nom duquel on agit. Or, si on fait de l'éducation à la citoyenneté un tel enjeu à l'école, et même tout au long de la vie, c'est qu'elle nous concerne tous et chacun au quotidien, dans notre vie partagée avec autrui. On souhaite que cette vie puisse être la meilleure possible pour soi, pour autrui, pour l'humanité, idéal jamais atteint et sans cesse à poursuivre et à promouvoir. En fonction de cet idéal et des orientations de l'éducation à la citoyenneté, on peut y rattacher les valeurs de liberté, d'égalité, de réciprocité, de démocratie et de concorde.

On peut certes dire que les valeurs de la démocratie et de la concorde sont à la base de l'idéal de citoyenneté. Or, la société doit pouvoir compter sur l'école pour former des démocrates, c'est-à-dire des citoyens qui prennent part aux débats et qui s'engagent dans la vie démocratique. Ensuite, la concorde s'avère un idéal à atteindre pour vivre ensemble, coopérer et se comprendre. Il s'agit d'apprendre à résoudre des conflits sans violence, sans domination, sans manipulation pour atteindre un bien précieux : la paix. Les valeurs de liberté, d'égalité et de réciprocité doivent être traitées ensemble. Reconnaître la liberté en soi et en autrui, voilà bien l'un des buts essentiels de toute éducation. C'est pourquoi on doit toujours la mettre en relation avec l'égalité et la réciprocité. Car la liberté de chacun doit rencontrer une limite : la liberté d'autrui. Si on a le droit de lutter pour sa liberté, les autres aussi ont ce droit. Nous ne pouvons demander aux autres de respecter notre liberté sans respecter la leur. Existe-t-il un lien entre ces valeurs et l'éducation mise en œuvre au jour le jour à l'école ? Autrement dit, y a-t-il une concordance entre les visées de l'éducation à la 
citoyenneté telle qu'elle se présente à la réflexion sur le sens de l'éducation et les pratiques enseignantes au quotidien de la vie en classe ?

\section{Certaines valeurs à la base de la pratique pédagogique}

Il est certes important d'analyser les orientations mises de l'avant pour l'éducation à la citoyenneté afin de dégager ses valeurs intrinsèques. L'histoire récente nous montre cependant qu'au nom de valeurs nobles comme la justice, l'égalité, l'indépendance, le droit des peuples, la démocratie, la lutte contre les privilèges, bien des atrocités ont été commises. Mais était-ce-ce réellement au nom de ces valeurs ou était-ce autre chose ? Toujours il faut aller voir au-delà des valeurs du discours et creuser pour dégager les valeurs qui supportent effectivement les gestes qu'on pose. Par exemple, dans les discours, nous sommes tous pour la démocratie : elle fonde l'école, on y apprend la démocratie et à devenir démocrates. Mais que faisons-nous concrètement pour la démocratie dans notre pratique pédagogique de tous les jours? Si la démocratie est si importante pour nous, la question à se poser c'est de se demander comment elle oriente et donne sens à notre pratique de tous les jours. On peut prendre toutes les valeurs de nos discours et poser les mêmes questions. On verra alors, selon une expression colorée d'administrateurs scolaires québécois, si nos bottines suivent nos babines. C'est avec ce rationnel que nous avons abordé des enseignantes et enseignants du secondaire pour leur demander de mettre en relief les valeurs concrètes qui orientent leur pratique pédagogique de tous les jours. C'est ainsi que 75 d'entre eux ont accepté d'identifier trois valeurs à la base de leur pratique pédagogique et d'illustrer concrètement leur actualisation. Plutôt que les 225 mentions attendues, nous en avons recueilli un total de 234 . Le tableau 1 montre la répartition des différentes valeurs identifiées.

Tableau 1: La répartition des valeurs identifiées

\begin{tabular}{|l|c|c|}
\hline Catégorie & $\begin{array}{l}\text { Nombre de valeurs } \\
\text { différentes }\end{array}$ & $\begin{array}{l}\text { Nombre } \\
\text { d'occurrences }\end{array}$ \\
\hline Valeurs morales & 13 & 23 \\
\hline Valeurs reliées au savoir & 15 & 33 \\
\hline $\begin{array}{l}\text { Valeurs reliées au développement } \\
\text { de la personne }\end{array}$ & 15 & 79 \\
\hline Valeurs sociales & 19 & 99 \\
\hline
\end{tabular}


Les répondantes et répondants n'avaient pas de liste de valeurs prédéfinies et devaient répondre de manière spontanée. Cela explique pourquoi un grand nombre de valeurs ont été identifiées une seule fois. Mais il n'en demeure pas moins que certaines valeurs se sont retrouvées souvent dans les réponses. Le tableau 2 donne une synthèse des valeurs le plus souvent identifiées.

Tableau 2 : Les valeurs le plus souvent identifiées

\begin{tabular}{|l|c|l|}
\hline Valeur & $\begin{array}{l}\text { Nombre } \\
\text { d'occurrences }\end{array}$ & Catégorie \\
\hline Respect & 42 & Sociale \\
\hline Autonomie & 39 & Personnelle \\
\hline Responsabilisation & 21 & Personnelle \\
\hline Participation & 17 & Sociale \\
\hline Sens des autres & 8 & Sociale \\
\hline Liberté & 7 & Morale \\
\hline Coopération & 6 & Sociale \\
\hline Communication & 6 & Sociale \\
\hline
\end{tabular}

Comme il s'agit d'enseignantes et enseignants du secondaire, on aurait pu s'attendre à plus de poids mis sur les valeurs reliées au savoir ou au développement intellectuel car ils se définissent beaucoup par la matière enseignée. Or, l'accent mis sur les valeurs personnelles et sociales illustre un souci de développement de la personne dans ses relations avec autrui. $\mathrm{Si}$ on compare les valeurs dégagées par les enseignantes et enseignants interrogés avec les valeurs qu'on trouve dans les orientations de l'éducation à la citoyenneté, les valeurs mises de l'avant vont dans le sens du développement du citoyen responsable personnellement, du citoyen capable de vivre avec les autres et participatif, mais moins sur le citoyen critique qui cherche la justice sociale.

\section{Conclusion}

Reconnaitre les valeurs profondes reliées à l'éducation à la citoyenneté peut nous aider à comprendre tout son sens et à identifier des pratiques qui la favorisent. L'éducation à la citoyenneté concerne bien sûr l'individu dans ses relations avec autrui dans l'espace commun que nous partageons tous. Elle commence peut-être dans des espaces restreints comme la classe, mais elle doit forcément s'ouvrir au milieu, à la communauté, à la planète. Elle commence peut-être aussi par la collaboration entre pairs, mais elle doit mener à la solidarité avec toutes les personnes qui partagent un même espace social. Il est certes important que l'éducation à la citoyenneté fasse partie du programme officiel. Mais les gestes que nous posons dans notre quotidien de pédagogues, des plus petits aux plus conscients en passant par les gestes inconscients, ce sont ceux-là qui, jour après jour, font œuvre d'éducation. Bien sûr, le curriculum officiel explicite doit orienter le travail de l'enseignante et de l'enseignant. Mais

20 Canadian and International Education Vol. 34 no. 1 - June 2005 
le curriculum implicite, ou curriculum caché, c'est lui qui marque profondément le parcours éducatif. C'est pourquoi on doit se demander comment les orientations et les valeurs de l'éducation à la citoyenneté atterrissent dans le concret des pratiques. Car ce qui reste lorsqu'on a tout oublié de notre passage dans les institutions socioéducatives, ce sont des attitudes, des savoir-être, des jugements sur ce qui est important et ce qui l'est moins, ce qui est acceptable et ce qui ne l'est pas. Chaque enseignante et chaque enseignant a donc le devoir de se demander, en réfléchissant à ses pratiques, lesquelles permettent réellement le développement de l'être humain soucieux d'autrui visant une vie bonne dans des institutions justes, idéal éthique explicité par Aristote encore et toujours d'actualité. Car l'éducation à la citoyenneté n'est pas seulement une forme d'éducation à la politique, elle est aussi porteuse d'une visée morale pour vivre ensemble.

\section{Notes bibliographiques}

Brossard, L. (1998). Contribuer à l'éducation à la citoyenneté dans toutes les disciplines. Vie pédagogique. 109, 25-34.

Bureau international de l'éducation (2003). Apprendre à vivre ensemble: avons-nous échoué? Synthèse des réflexions et des contributions issues de la 46è session de la conférence internationale de l'éducation de l'Unesco tenue à Genève du 5 au 8 septembre 2001. Genève : BIE et UNESCO.

Conseil supérieur de l'éducation (1998). Éduquer à la citoyenneté. Rapport annuel sur l'état et les besoins de l'éducation. Sainte-Foy : Conseil supérieur de l'éducation.

Gouvernement du Québec (2003). Programme de formation de l'école québécoise: Enseignement secondaire 1er cycle. Québec: Ministère de l'Éducation du Québec.

Gouvernement du Québec (1997). Le virage du succès. Québec: Ministère de l'Éducation du Québec.

Gouvernement du Québec (1996). Rénover notre système d'éducation : dix chantiers prioritaires. Rapport final de la Commission des États généraux sur l'éducation. Québec : Ministère de l'Éducation du Québec.

Guay, L. \& Jutras, F. (2004). La formation initiale et continue des maîtres en éducation à la citoyenneté. In F. Ouellet (dir.) Quelle formation pour l'éducation à la citoyenneté? (pp. 195-208). Sainte-Foy : PUL

Jutras, F. \& Boudreau, C. (1997). La dimension éthique dans la relation éducative selon le point de vue d'enseignantes et enseignants du secondaire. In M. P. Desaulniers, F. Jutras, G.A. Legault \& P. Lebuis (dir.), Les défis éthiques en éducation (pp. 155-169). Sainte-Foy : PUQ.

Meirieu, P. (1997). L'éducation aux droits de l'homme : quelques jalons, valeurs et pistes d'action. In J. Hénaire (dir.), Droits de l'homme et citoyenneté : des repères pour agir (pp. 107-120). Genève : Centre international de formation à l'enseignement des droits de l'homme et de la paix (Collection thématique, $\mathrm{n}^{\circ} 5$ ).

Mintrop, H. (2002). Teachers and civic education instruction in cross-national comparison. International Perspectives on Education and Society. 5, 61-83.

Munger, M. (2004). Nouveau record de faible participation. La Presse. 30 juin, A6.

Osler, A. \& Starkey, H. (1997). Enjeux de l'éducation à la citoyenneté démocratique pour la formation des maîtres. In J. Hénaire (dir.), Droits de l'homme et citoyenneté : 
des repères pour agir (121-135). Genève : Centre international de formation à l'enseignement des droits de l'homme et de la paix (Collection thématique, no 5).

Pagé, M. (2001). L'éducation à la citoyenneté devant la diversité des conceptions de la citoyenneté. In M. Pagé, F. Ouellet \& L. Cortesao (dir.) L'éducation à la citoyenneté (pp. 41-54). Sherbrooke : CRP.

Reboul, O. (1989). La philosophie de l'éducation. Paris : PUF, collection QSJ.

Soares, S.R. (2004). Étude des représentations de la citoyenneté chez des professeurs formateurs de futurs enseignants dans une université publique de l'État de Bahia au Brésil. Thèse de doctorat, Faculté d'éducation, Université de Sherbrooke, Sherbrooke.

UNESCO (1996). L'éducation : un trésor est caché dedans. Rapport à l'UNESCO sur l'éducation pour le vingt et unième siècle, Commission présidée par Jacques Delors. Paris : UNESCO et Odile Jacob. 